University of Warwick institutional repository: http://go.warwick.ac.uk/wrap This paper is made available online in accordance with publisher policies. Please scroll down to view the document itself. Please refer to the repository record for this item and our policy information available from the repository home page for further information.

To see the final version of this paper please visit the publisher's website. Access to the published version may require a subscription.

Author(s): D. J. Paul, J. R. A. Cleaver, H. Ahmed, and T. E. Whall Article Title: Coulomb blockade in silicon based structures at temperatures up to $50 \mathrm{~K}$ Year of publication: 1994

Link to published version: http://dx.doi.org/10.1063/1.109972

Publisher statement: none 


\title{
Coulomb blockade in silicon based structures at temperatures up to $50 \mathrm{~K}$
}

\author{
D. J. Paul, J. R. A. Cleaver, and H. Ahmed \\ Microelectronics Research Centre, Cavendish Laboratory, Cambridge University, Madingley Road, \\ Cambridge CB3 OHE, United Kingdom \\ T. E. Whall \\ Department of Physics, University of Warwick, Coventry CV4 7AL, United Kingdom
}

(Received 22 March 1993; accepted for publication 26 May 1993)

Coulomb blockade has been observed in the current-voltage characteristics of structures fabricated in silicon germanium $\delta$-doped material at temperatures up to $50 \mathrm{~K}$. This is consistent with the estimated effective tunnel capacitance of $10 \mathrm{aF}$ which is significantly smaller than the reported capacitances of tunnel junctions made from $\mathrm{Al}$ or GaAs/AlGaAs heterostructures.

In the past few years, technological advances in lithography, etching, and crystal growth have enabled the fabrication of tunnel junctions with capacitances small enough to observe single electron tunneling (SET) effects. These effects occur when the elementary charging energy, $e^{2} / 2 C$, of the junction capacitance $C$, is larger than the energy of the thermal fluctuations, $k_{B} T$, and the tunnel resistance is larger than the resistance quantum $h / e^{2}(\approx 26 \mathrm{k} \Omega)$ to avoid smearing by quantum fluctuations. Here, $e$ is the absolute value of the electron charge, $k_{B}$ is the Boltzmann constant, $T$ is the absolute temperature, and $h$ is Planck's constant. At voltages less than $e / 2 C$, electron tunneling is suppressed and Coulomb blockade is observed. ${ }^{1}$ Devices using SET effects such as the single electron transistor, ${ }^{2}$ turnstile, electron pump, ${ }^{3}$ and the single electron memory ${ }^{4}$ have been demonstrated.

The realization of ultrasmall tunnel junctions has been achieved by a number of different fabrication techniques including double angle evaporation of $\mathrm{Al}$, Schottky gate confinement of the two-dimensional electron gas (2DEG) formed at $a$ heterointerface between $\mathrm{GaAs}$ and $\mathrm{Al}_{x} \mathrm{Ga}_{1-x}$ As (Ref. 3), and sidegated ${ }^{5}$ confinement of a 2DEG in $\delta$-doped GaAs. ${ }^{6}$ While GaAs is the predominant semiconducting material used for investigating quantum and SET effects, the vast majority of all semiconductor production is in silicon. The use of silicon germanium epitaxial layers on silicon can expand the use of silicon by introducing band-gap engineering, strain engineering, higher mobility, and the possibility of direct or pseudodirect band-gap material. ${ }^{7}$ In the present work, we have investigated the possibilities of single electron effects in this material system. While Coulomb blockade has previously been observed in Ge particles in a Si matrix, ${ }^{8}$ the random distribution of the particles is not ideal for device fabrication.

Electron beam lithography and reactive ion etching (RIE) have been used to fabricate structures in silicon germanium $\delta$-doped material. The $\delta$-doped material was grown by molecular-beam epitaxy on a $\mathrm{Si}(100) n^{-}$substrate; an undoped silicon layer followed by a strained, 50-nm-thick epitaxial layer of undoped $\mathrm{Si}_{0.8} \mathrm{Ge}_{0.2}$, a boron $\delta$ layer, and finally a 50-nm-thick capping layer of undoped $\mathrm{Si}_{0.8} \mathrm{Ge}_{0.2}$. Measurements on a Hall bar sample at $4.2 \mathrm{~K}$ indicate a carrier concentration of $2 \times 10^{18} \mathrm{~m}^{-2}$ and a mobility of $0.003 \mathrm{~m}^{2} / \mathrm{V} \mathrm{s}$. Optical lithography was used for mesa isolation and ohmic contact fabrication. Aluminum (1\% silicon) ohmic contacts were annealed in an electron beam rapid thermal annealer. Electron beam lithography at $60 \mathrm{kV}$ was used to define wires and sidegates in 100-nmthick polymethylmethacrylate (PMMA) resist developed in a 3:1 solution of isopropyl-alcohol:methyl-isobutylketone for $30 \mathrm{~s}$ before a $20 \mathrm{~s} \mathrm{O}_{2}$ plasma etch and $20 \mathrm{~nm}$ of nickel-chromium ( $\mathrm{NiCr})(80 \%-20 \%)$ was evaporated and lifted off. The NiCr was used as a RIE mask to define small features while bond pads were protected by optical resist. All RIE was performed on a STS 320PC parallel plate etcher operating at $300 \mathrm{~W}, 13.56 \mathrm{MHz}$ at a pressure of 20 mTorr with flow rates of 20 standard cubic centimeters per minute $\left(\mathrm{sccm}\right.$ ) of $\mathrm{SiCl}_{4}$, and $20 \mathrm{sccm}$ of $\mathrm{CF}_{4}$ for 90 s. ${ }^{9}$ This produces an etch depth of approximately $150 \mathrm{~nm}$. A structure consisting of a wire and a sidegate with a small island between them was fabricated (Fig. 1). The channel and sidegate are $\delta$-doped $\mathrm{Si}_{0.8} \mathrm{Ge}_{0.2}$ but the exact structure of the island is not clear from scanning electron microscope (SEM) pictures.

Clear Coulomb blockade effects including the Coulomb staircase have been observed at $4.2 \mathrm{~K}$ using standard $\mathrm{dc}$ four terminal measurements between the wire and the sidegate (Fig. 2). Previous observations in $\delta$-doped material have only reported Coulomb blockade at $30 \mathrm{mK}$ (Ref.

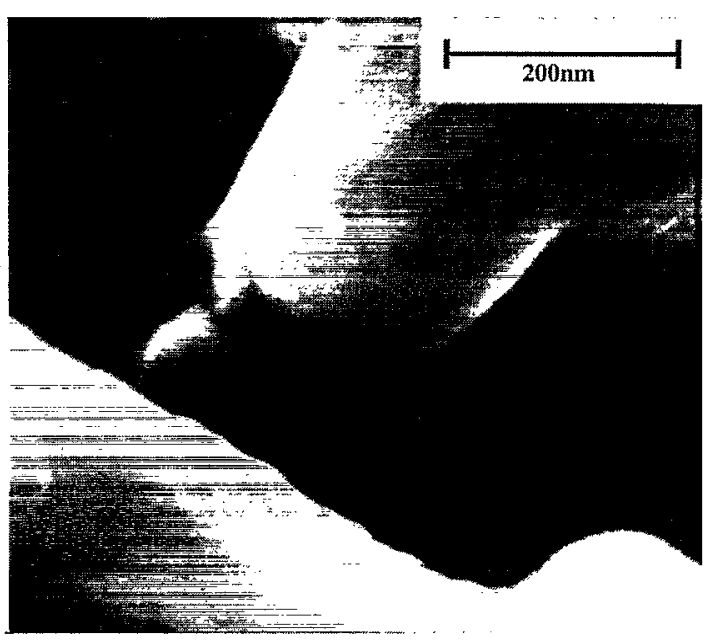

FIG. 1. Scanning electron microscope picture (at $30^{\circ}$ tilt) of the $50-\mathrm{nm}^{-}$ wide island between the wire and sidegate. 


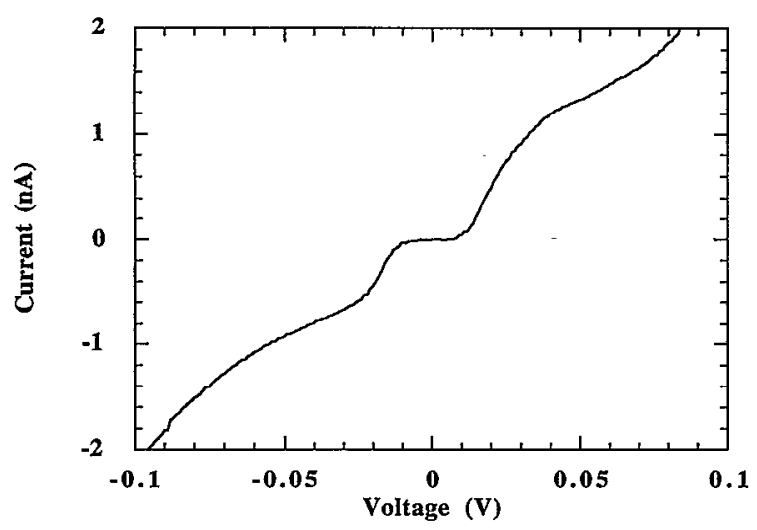

FIG. 2. dc current-voltage measurement at $4.2 \mathrm{~K}$ between the wire and sidegate showing clear Coulomb blockade.

4) and $300 \mathrm{mK} .{ }^{6}$ The total capacitance of the tunnel junctions was estimated to be approximately $10 \mathrm{aF}$ from the Coulomb gap, $e / 2 C \approx 8 \mathrm{mV}$ at $1.7 \mathrm{~K}$. This value of capacitance predicts a transition temperature of around $92 \mathrm{~K}$ when the SET effects should be smeared out by thermal fluctuations. The sum of the tunnel resistances was estimated at $11 \mathrm{M} \Omega\left(\gg h / e^{2}\right)$ from the current-voltage characteristics. At temperatures above liquid helium, the blockade was observed with zero current plateau up to $15 \mathrm{~K}$ (Fig. 3). Above $15 \mathrm{~K}$ the plateau began to tilt as expected as the probability of thermally induced tunneling increases. By $50 \mathrm{~K}$ the current-voltage characteristic was still nonlinear around $0 \mathrm{~V}$ but SET effects were almost completely washed out by thermal fluctuations (Fig. 3).

A comparison can be made between the capacitance given by the Coulomb gap and the estimated selfcapacitance of the island by considering the selfcapacitance $\left(C_{0}\right)$ with respect to infinity of a disk of radius $r$, for which $C_{0}=8 \varepsilon_{,} \varepsilon_{0} r$. The relative dielectric constant $\left(\varepsilon_{r}\right)$ used in such a calculation must represent both the vacuum gap and the effects of substrate coupling that are observed in sidegated structures. ${ }^{5}$ It can be estimated

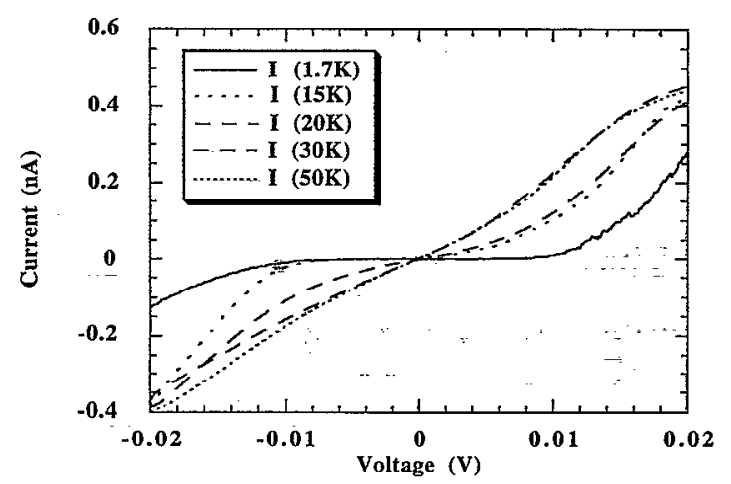

FIG. 3. Temperature dependence of the current-voltage characteristics around $0 \mathrm{~V}$. roughly as the mean of the relative dielectric constants for vacuum and the substrate. The width of the Coulomb gap gives a capacitance of about $10 \mathrm{aF}$ and by considering 10 $\mathrm{aF}$ as the self-capacitance a radius of $22 \mathrm{~nm}$ is produced by the above formula. The radius of the island from SEM pictures is $25 \mathrm{~nm}$. This shows good agreement especially when the sidewall depletion width due to RIE is considered. This is known to be small in GaAs $\delta$-doped material ${ }^{5}$ with a carrier concentration of about $10^{17} \mathrm{~m}^{-2}$ compared to $2 \times 10^{18} \mathrm{~m}^{-2}$ in the present silicon germanium material. The depletion width has not been measured in $\delta$-doped silicon germanium but is expected to be similar.

The small size of the dot precludes the unambiguous identification of the dot material as $\delta$-doped SiGe. Magnetoresistance measurements suggest that the $\mathrm{NiCr}$ layer has frozen out and plays no part in the observed effects; 300 $\mathrm{nm}$ wide wires in the same material fabricated both with and without the $\mathrm{NiCr}$ etch mask show no difference in magnetoresistance when measured at liquid helium temperatures. Surface states, however, may contribute to the observed effects. Measurements on $\mathrm{Si} / \mathrm{SiGe} 2 \mathrm{DEG}$ structures ${ }^{10}$ indicate a relatively high surface state density of $1-2 \times 10^{16} \mathrm{~m}^{-2}$; their exact position in the band gap has not been measured but will be closer to the conduction band than the valence band. Hence tunneling through surface states may contribute to the observed effects.

In conclusion, single electron effects have been observed in silicon germanium $\delta$-doped material. Coulomb blockade was observed at temperatures up to $50 \mathrm{~K}$ from a structure acting as an island of charge with a capacitance of about $10 \mathrm{aF}$. The observations confirm that islands with smaller sizes could allow the realization of a single electron transistor working at liquid nitrogen or higher temperatures.

The authors wish to thank S. Blythe for help with the RIE. D.J.P. acknowledges the SERC (CASE) studentship associated with BNR. This work was carried out under the Silicon Towards 2000 scheme of the DTI.

${ }^{1}$ D. V. Averin and K. K. Likharev, in Mesoscopic Phenomena in Solids, edited by B. L. Altshuler, P. A, Lee, and R. A. Webb (North-Holland, Amsterdam, 1991), p. 169.

${ }^{-2}$ T. A. Fulton and G. J. Dolan, Phys. Rev. Lett. 59, 109 (1987).

${ }^{3}$ M. H. Devoret and H. Grabert, in Single Charge Tunneling, edited by

H. Grabert and M. H. Devoret (Plenum, New York, 1992), p. 1.

${ }^{4}$ K. Nakazato, R. J. Blaikie, J. R. A. Cleaver, and H. Ahmed, Electron. Lett. 29, 384 (1993).

${ }^{5}$ Y. Feng, T. J. Thornton, J. J. Harris, and D. A. Williams, Appl. Phys. Lett. 60, 94 (1992).

${ }^{6}$ K. Nakazato, T. J. Thornton, J. White, and H. Ahmed, Appl. Phys. Lett. 61, 3145 (1992).

${ }^{7}$ E. Kasper and F. Schäffler, in Semiconductors and Semimetals, edited by T. P. Pearsall (Academic, San Diego, 1991), Vol. 33, p. 223.

${ }^{8}$ A. I. Yakimov, V. A. Markov, A. V. Dvurechenskii, and O. P. Pchelyakov, Philos. Mag. B 65, 701 (1992).

${ }^{9}$ D. J. Paul, J. R. A. Cleaver, and H. Ahmed, Microelectron. Eng. 21, 349 (1993).

${ }^{10}$ K. Ismail, B. S. Meyerson, and P. J. Wang, Appl. Phys. Lett. 58, 2117 (1991). 